\title{
Quantification of STEM-EDS With Ion Implantation
}

\author{
R. Garcia, Y. Liu, F. A. Stevie
}

Analytical Instrumentation Facility, North Carolina State University, Raleigh, NC 27695, USA

Energy dispersive spectroscopy (EDS) is widely used to provide elemental analysis on SEM and (S)TEM instruments. The detection limit is commonly quoted as approximately $1 \%$ atomic and determined from analysis of high purity specimens of the element of interest or estimated by theoretical calculations. Earlier studies [1-3] showed that high dose ion implantation could be used to quantify and obtain a detection limit for STEM-EDS analyses on a cross section specimen from a silicon substrate. The procedure is to obtain a lift-out TEM cross section specimen [4] from a sample that has been ion implanted with sufficient dose to be detected with EDS. Since the sample is very thin the EDS can have high lateral resolution. A line scan starting from the surface will provide a concentration profile of the implanted species. For an implant dose of $1 \times 10^{16}$ atoms $/ \mathrm{cm}^{2}$, the peak concentration will be approximately $1 \times 10^{21}$ atoms $/ \mathrm{cm}^{3}$. For a silicon substrate with density $5 \times 10^{22}$ atoms $/ \mathrm{cm}^{3}$ this concentration is $2 \%$ atomic which is above the stated detection limit. Accurate concentrations can be established by measurement of dose with Rutherford Backscattering Spectroscopy and depth profile analysis with SIMS. [5,6]

Instrumentation has shown significant development since the earlier work and it was of interest to perform a similar analysis on an instrument with current EDS capability. Specimens ware prepared using an in-situ lift-out method [4] on a FEI Quanta 3D FIB-SEM. Images of the nickel implanted TEM specimen cut away from the silicon substrate and attached to a TEM grid are shown in Figure 1 A) and B) respectively. Data were obtained for samples separately implanted with $1 \times 10^{16}$ atoms $/ \mathrm{cm}^{2}$ of nickel and cobalt, both at $100 \mathrm{keV}$.

EDS analysis was made at $200 \mathrm{keV}$ on a FEI Titan 80-300 aberration corrected STEM with a SuperX EDS system that uses four Bruker silicon drift detectors and has energy resolution of $136 \mathrm{eV}$. Counts were obtained at $1 \mathrm{~nm}$ intervals and raw counts for the nickel implanted specimen are shown in Fig. 2. The data accumulation time was $22 \mathrm{~min}$. Fig. 3 displays a SIMS depth profile and a five point moving average of the nickel $\mathrm{K}$ line EDS line scan normalized to match the peak with the SIMS profile. The five point average plot was selected to approximate the data obtained if $5 \mathrm{~nm}$ steps were used for data acquisition instead of $1 \mathrm{~nm}$. The nickel detection limit is approximately $0.2 \%$ atomic. Analysis of the cobalt sample showed the same detection limit.

Analyses made on identical samples in 2002 indicated detection limits of $0.2 \%$ atomic for the nickel $\mathrm{K}$ line measurement and $0.25 \%$ atomic for the cobalt $\mathrm{K}$ line scan. The results show a detection limit below $1 \%$ atomic can be achieved. Besides a detection limit these results provide a mechanism for accurate EDS quantification at high lateral resolution. Inhomogeneous samples, such as minerals, should be quantifiable with this method because the calibration implant would put the same concentration into the entire sample. If the concentration of the species of interest is too high in the sample, then a rare isotope, such as ${ }^{15} \mathrm{~N}$ instead of ${ }^{14} \mathrm{~N}$, may be implanted. It should also be noted that the EDS counts for a matrix element, in this case silicon, can be used to rapidly determine the thickness of a specimen. Samples with a known thickness would be used to calibrate the EDS counts. 


\section{References:}

[1] C. B. Vartuli et al, Microscopy and Microanalysis Proceedings Vol. 6, Suppl. 2 (2000) p. 536.

[2] C. B. Vartuli et al, Microscopy and Microanalysis Proceedings (2001) p. 200.

[3] C. B. Vartuli et al, Microscopy and Microanalysis Proceedings (2002) 8 (Suppl. 2) p. 1192CD. [4] L. A. Giannuzzi et al, in "Introduction to Focused Ion Beams" eds. L. A. Giannuzzi and F. A. Stevie (Springer, New York 2005) p. 201.

[5] R. G. Wilson, F. A. Stevie, and C. W. Magee "Secondary Ion Mass Spectrometry" (Wiley, New York 1989) Section 3.1

[6] F. A. Stevie "Secondary Ion Mass Spectrometry” (Momentum Press, New York 2016) Chapter 6.

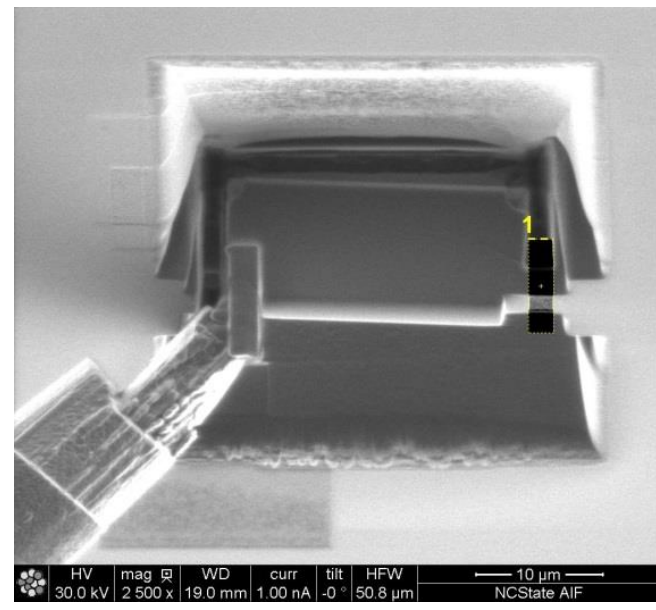

A)

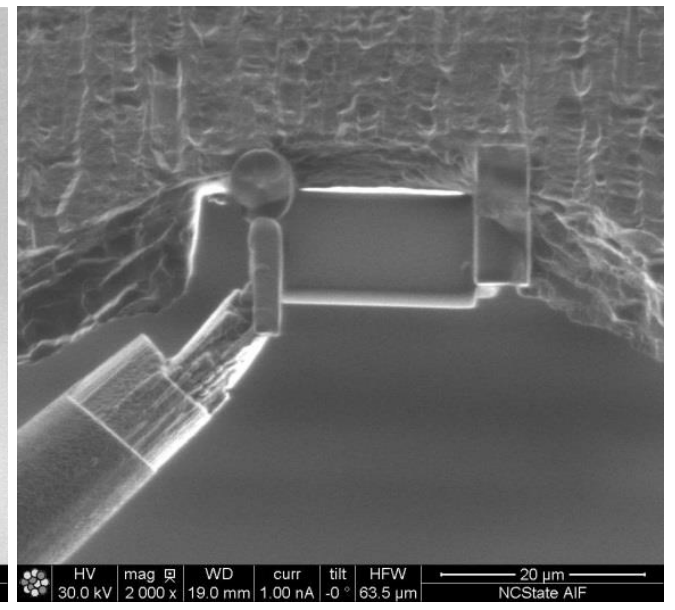

B)

Figure 1. Lift-out Ni specimen A) cut away from substrate and B) attached to TEM grid

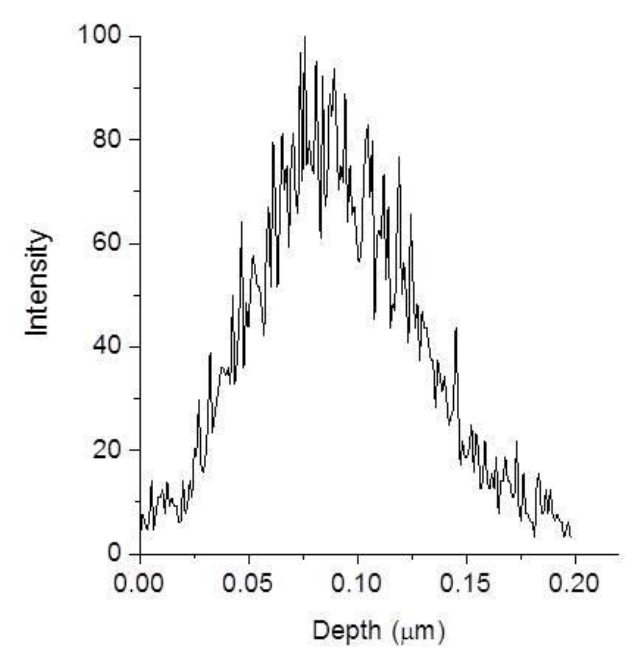

Figure 2. EDS line scan of Ni specimen

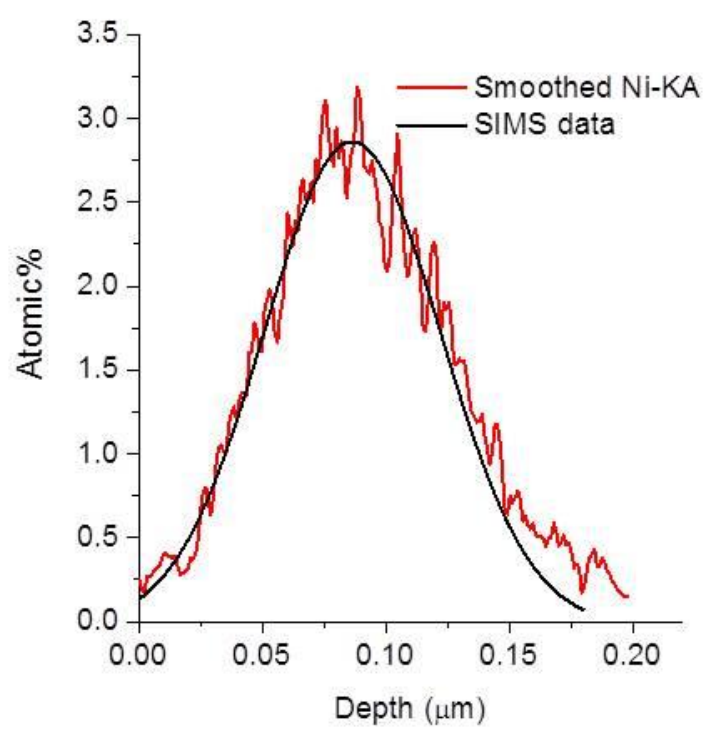

Figure 3. SIMS and EDS of Ni specimen 